\title{
Multiheme hydroxylamine oxidoreductases produce NO during ammonia oxidation in methanotrophs
}

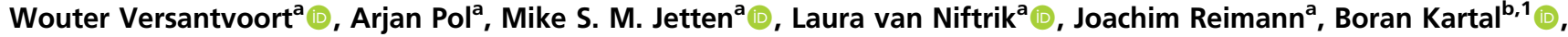 \\ and Huub J. M. Op den Camp ${ }^{a}$ (1) \\ ${ }^{a}$ Department of Microbiology, Institute for Water and Wetland Research, Faculty of Science, Radboud University, 6525 AJ Nijmegen, The Netherlands;
and b Microbial Physiology Group, Max Planck Institute for Marine Microbiology, 28359 Bremen, Germany
}

Edited by Caroline S. Harwood, University of Washington, Seattle, WA, and approved August 6, 2020 (received for review June 2, 2020)

\begin{abstract}
Aerobic and nitrite-dependent methanotrophs make a living from oxidizing methane via methanol to carbon dioxide. In addition, these microorganisms cometabolize ammonia due to its structural similarities to methane. The first step in both of these processes is catalyzed by methane monooxygenase, which converts methane or ammonia into methanol or hydroxylamine, respectively. Methanotrophs use methanol for energy conservation, whereas toxic hydroxylamine is a potent inhibitor that needs to be rapidly removed. It is suggested that many methanotrophs encode a hydroxylamine oxidoreductase (mHAO) in their genome to remove hydroxylamine, although biochemical evidence for this is lacking. HAOs also play a crucial role in the metabolism of aerobic and anaerobic ammonia oxidizers by converting hydroxylamine to nitric oxide (NO). Here, we purified an HAO from the thermophilic verrucomicrobial methanotroph Methylacidiphilum fumariolicum SolV and characterized its kinetic properties. This mHAO possesses the characteristic $\mathbf{P}_{\mathbf{4 6 0}}$ chromophore and is active up to at least $80{ }^{\circ} \mathrm{C}$. It catalyzes the rapid oxidation of hydroxylamine to NO. In methanotrophs, mHAO efficiently removes hydroxylamine, which severely inhibits calcium-dependent, and as we show here, lanthanidedependent methanol dehydrogenases, which are more prevalent in the environment. Our results indicate that mHAO allows methanotrophs to thrive under high ammonia concentrations in natural and engineered ecosystems, such as those observed in rice paddy fields, landfills, or volcanic mud pots, by preventing the accumulation of inhibitory hydroxylamine. Under oxic conditions, methanotrophs mainly oxidize ammonia to nitrite, whereas in hypoxic and anoxic environments reduction of both ammonia-derived nitrite and NO could lead to nitrous oxide $\left(\mathrm{N}_{2} \mathrm{O}\right)$ production.
\end{abstract}

hydroxylamine oxidoreductase | ammonia oxidation | methanotrophy multiheme cytochrome | nitric oxide

$\mathbf{M}$ ethane $\left(\mathrm{CH}_{4}\right)$ is a potent greenhouse gas with a global warming potential 34 times that of carbon dioxide $\left(\mathrm{CO}_{2}\right)$ over a 100-y time span (1). Microbial anaerobic degradation of biomass yields organic acids and hydrogen that fuel methanogenesis. Methanogenic archaea are responsible for the production of the majority of biogenic methane (about $583 \mathrm{Tg} \cdot \mathrm{y}^{-1}$ ) (2), which is subsequently released to the environment. Emission of this methane to the atmosphere is partly mitigated by aerobic and anaerobic methanotrophs (3). These microorganisms use methane as an energy source by oxidizing it to $\mathrm{CO}_{2}$ and act as a methane sink, thereby reducing the contribution of methane on global warming. On the other hand, aerobic and nitrite-dependent methanotrophs can be the source of another, even more potent greenhouse gas, nitrous oxide $\left(\mathrm{N}_{2} \mathrm{O}\right)$, which has a global warming potential 296 times that of $\mathrm{CO}_{2}$ over a 100 -y time span $(1,4)$. According to the Fifth Assessment Report of the Intergovernmental Panel on Climate Change, the total radiative forcing of $\mathrm{CH}_{4}$ and $\mathrm{N}_{2} \mathrm{O}$ increased by $2 \%$ and $6 \%$, respectively, since the previous report (1). This increase makes $\mathrm{N}_{2} \mathrm{O}$ the third largest contributor to radiative forcing. Furthermore, since the chlorofluorocarbons were phased out of use, $\mathrm{N}_{2} \mathrm{O}$ became the primary ozone-depleting greenhouse gas (5). Consequently, methanotrophs sit in the nexus of both carbon and nitrogen cycles and can act both as sources and sinks of climate active gases. Therefore, understanding their metabolism and how this is influenced by anthropogenic activities will help us predict their effect on global warming and possibly utilize them to counteract emissions (6-10).

Ammonia oxidation in the natural environment has been mainly attributed to a distinct group of microorganisms, termed ammonia oxidizers $(11,12)$. However, in natural and engineered environments where methane and ammonia co-occur, methanotrophs can also contribute significantly to ammonia oxidation $(8,9,13-17)$.This cross-reactivity is due to the structural similarity between methane and ammonia, which allows both methanotrophs and ammonia oxidizers to convert either substrate, although neither is capable of growing on the alternative substrate $(4,9,17)$. The first step in both methane and ammonia oxidation is the incorporation of oxygen by methane/ ammonia monooxygenase (MMO, AMO), resulting in the formation of methanol or hydroxylamine, respectively $(4,17-19)$. In methane-oxidizing bacteria, methanol dehydrogenase (MDH) converts methanol to formaldehyde (or formate), which is further oxidized to $\mathrm{CO}_{2}(6,20,21)$. Whereas in ammonia-oxidizing microorganisms, hydroxylamine oxidoreductase (HAO) oxidizes hydroxylamine to nitric oxide (NO), which is subsequently converted to nitrite (22-24). In octaheme HAO proteins catalysis occurs at heme 4 , which is cross-linked to a tyrosine residue from a neighboring subunit, covalently linking all three subunits of the HAO complex $(22,25-29)$. This cross-link forces the heme into a

\section{Significance}

Methanotrophs oxidize methane to $\mathrm{CO}_{2}$, thereby mitigating the emission of this potent greenhouse gas. Understanding how these microorganisms are influenced by anthropogenic activities will help better predict their impact on global warming and utilize them to reduce it. Ammonia-fertilizer input to the environment has greatly improved crop yields but increased emissions of greenhouse gasses like $\mathrm{N}_{2} \mathrm{O}$ as well. In methane-rich environments, methanotrophs play a prominent role in ammonia oxidation. Here, we purified a protein (mHAO) from Methylacidiphilum fumariolicum, capable of rapid oxidation of hydroxylamine to NO. We propose that mHAO enables methanotrophs to cope with high ammonia concentrations, leading to reduced methane emissions. However, this activity simultaneously contributes to ammonia loss and nitrite production, and potentially leads to $\mathrm{N}_{2} \mathrm{O}$ emissions.

Author contributions: W.V., A.P., J.R., B.K., and H.J.M.O.d.C. designed research; W.V. and A.P. performed research; W.V., A.P., M.S.M.J., L.v.N., J.R., B.K., and H.J.M.O.d.C. analyzed data; and W.V. and B.K. wrote the paper.

The authors declare no competing interest.

This article is a PNAS Direct Submission.

Published under the PNAS license.

${ }^{1}$ To whom correspondence may be addressed. Email: bkartal@mpi-bremen.de.

This article contains supporting information online at https://www.pnas.org/lookup/suppl/ doi:10.1073/pnas.2011299117/-/DCSupplemental.

First published September 10, 2020. 
highly ruffled conformation, giving rise to the characteristic absorbance peak at $460 \mathrm{~nm}\left(\mathrm{P}_{460}\right)$ in the reduced enzyme $(26,27)$. The active site of HAOs strongly favors oxidative reactions and the cross-linking tyrosine is highly conserved within members of the HAO family (28).

Hydroxylamine is a highly toxic compound and has been shown to inhibit calcium-dependent MDHs (30), which necessitates the rapid turnover of hydroxylamine in methane-oxidizing bacteria. Many methanotrophs encode a HAO-like protein (mHAO) homologous to that of bacterial ammonia oxidizers. These HAO-like proteins have been postulated to perform hydroxylamine oxidation in methanotrophs, but biochemical evidence for this is lacking (4). Up-regulation of hao transcription in response to ammonia has been shown for several methanotrophic bacteria that oxidize ammonia faster compared to the ones not encoding hao genes (31-34). Most cultured methanotrophs oxidize ammonium to nitrite $(17,33-35)$, whereas a few methanotrophs have been shown to produce $\mathrm{N}_{2} \mathrm{O}$ from ammonia oxidation without apparent nitrite production (36). In addition, $\mathrm{N}_{2} \mathrm{O}$ production has been observed for many aerobic methanotrophs under nitrite-reducing conditions, either as a product of reactive nitrogen detoxification or respiration under $\mathrm{O}_{2}$ limitation $(31,34,36-38) . \mathrm{N}_{2} \mathrm{O}$ production from ammonia and hydroxylamine is thought to occur via the initial formation of nitrite, which until recently was the presumed end product of hydroxylamine oxidation by all HAOs. In this hypothesis, nitrite reductases nirS and nirK would produce NO, which would subsequently be reduced to $\mathrm{N}_{2} \mathrm{O}$ by a nor $B C$ complex $(29,31-34)$. However, the close amino acid sequence similarity of the mHAO proteins to those encoded by ammonia-oxidizing bacteria suggests that the end product of mHAO could also be NO $(27,28)$. Thus, NO would be produced as an obligate intermediate in ammonia oxidation by methanotrophs as well.

To date, there is no biochemical evidence for the role of these HAO-like proteins in hydroxylamine oxidation in methanotrophic bacteria. Here, we present the purification of an mHAO protein from the verrucomicrobial thermoacidiphilic methanotroph Methylacidiphilum fumariolicum SolV (39). We studied the catalytic properties of this methanotroph HAO and compared it to its homologs from both aerobic and anaerobic ammonia oxidizers. We suggest that mHAO has a crucial role in preventing the hydroxylamine inhibition of $\mathrm{MDH}$, and help methanotrophs thrive in environments where methane and ammonia coexist.

\section{Results}

mHAO Catalyzes the Oxidation of Hydroxylamine to NO. $M$. fumariolicum SolV encodes an HAO-like protein with an $\mathrm{N}$-terminal fusion to a monoheme cytochrome $c$ in its genome (haoA, Mfumv2_2472). The molecular mass of a single subunit of this protein is $83,860 \mathrm{Da}$ including the protein backbone and 9 heme $c$ moieties, excluding the signal peptide. Multiple sequence alignment of this protein with other HAO-like proteins from both aerobic and anaerobic ammonia oxidizers and methanotrophs (SI Appendix, Fig. S1) showed that heme spacing and the cross-linking tyrosine were conserved among all analyzed proteins. This tyrosine (TYR467, numbering of NeHAO) (26) covalently binds the three HAO monomers. To examine whether this HAO-like protein from M. fumariolicum SolV is a bona fide HAO, capable of oxidizing hydroxylamine, the enzyme was purified to homogeneity by a two-step column chromatography procedure. As observed for HAO-like proteins with the tyrosine cross-link $(27,40)$, the purified enzyme showed two bands on a denaturing SDS/PAGE, both of which had a dominant heme stain signal (SI Appendix, Fig. S2A). Both SDS/PAGE bands were identified as the gene product of Mfumv2_2472 (haoA) by matrix-assisted laser desorption/ionization time-of-flight mass spectrometry (MALDITOF MS). On a nondenaturing clear native PAGE, a band at $500 \mathrm{kDa}$ and a band at $250 \mathrm{kDa}$ were observed (SI Appendix, Fig. $\mathrm{S} 2 B$ ). Both bands were stained with an in-gel hydroxylamine

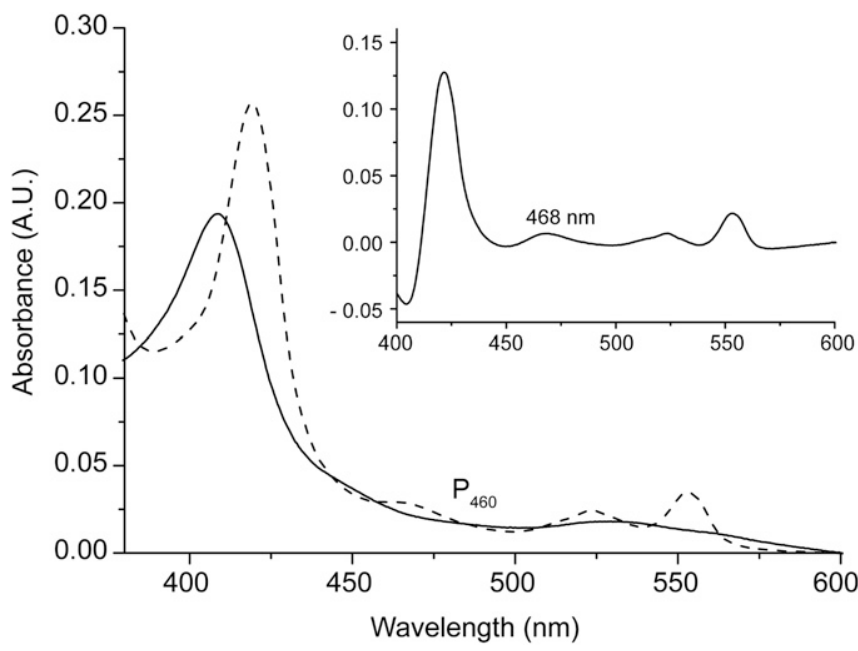

Fig. 1. UV-vis absorbance spectrum of Methylacidiphilum fumariolicum SolV HAO in the as-isolated (solid) and dithionite reduced state (dashed). Inset shows the reduced minus oxidized difference spectrum. The $P_{460}$ chromophore characteristic for HAOs is clearly present in both the reduced and reduced minus oxidized difference spectrum.

oxidation stain. The UV-vis spectrum of purified mHAO displayed a prominent maximum at $468 \mathrm{~nm}$ in the reduced form. In addition, characteristic heme $c$ features, namely a maximum in the Soret region at $408 \mathrm{~nm}$ in the oxidized form (Fig. 1, solid) and maxima at $418 \mathrm{~nm}$ for the Soret and 522 and $551 \mathrm{~nm}$ for the beta and alpha bands, respectively (Fig. 1, dashed) were observed. Activity assays with bovine cytochrome $c$ as electron acceptor showed that mHAO catalyzed the rapid oxidation of hydroxylamine with a $V_{\max }$ of $5.5 \pm$ $0.1 \mu \mathrm{mol} \cdot \mathrm{min}^{-1} \cdot \mathrm{mg}$ protein ${ }^{-1}, K_{\mathrm{m}}$ of $1.4 \pm 0.1 \mu \mathrm{M}$, and a $K_{\text {cat }}$ of $7.2 \pm 0.15 \mathrm{~s}^{-1}$ (Table 1).

To determine the end-product of hydroxylamine oxidation by mHAO, two different methods were employed. First, the production of $\mathrm{NO}$ from hydroxylamine by mHAO was examined using membrane inlet mass spectrometry (MIMS). In the absence of oxygen, mHAO catalyzed the stoichiometric conversion of hydroxylamine to $\mathrm{NO}$ with a recovery rate of $94 \pm 4 \%$ of the added hydroxylamine as NO (Fig. $2 A$ ). The production of NO was corroborated by following the reduction of bovine cytochrome $c$ by mHAO with a limiting amount of hydroxylamine (Fig. $2 B$ ). Here, the electron stoichiometry of hydroxylamine oxidation was determined to be 3 to $1(3 \pm 0.06 \mu \mathrm{M}$ of reduced cytochrome $c$ per $\mu \mathrm{M}$ hydroxylamine) indicating the formation of NO (Eq. 1). These results were in line with recent studies that established NO to be the product of hydroxylamine oxidation by HAO enzymes $(27,29)$ :

$$
\mathrm{NH}_{2} \mathrm{OH} \rightarrow \mathrm{NO}+3 e^{-}+3 \mathrm{H}^{+} .
$$

mHAO Is Tuned to Higher Temperature. The temperature dependence of hydroxylamine oxidation by mHAO and its homolog from the anaerobic ammonium oxidizing bacterium Kuenenia stuttgartiensis (KsHAO) were investigated in the range of 20 to $80^{\circ} \mathrm{C}$ (Fig. 3). KsHAO showed a temperature optimum at $50{ }^{\circ} \mathrm{C}$ and was no longer active at temperatures above $60{ }^{\circ} \mathrm{C}$. mHAO showed a temperature optimum at $60{ }^{\circ} \mathrm{C}$ and maintained $\sim 80 \%$ of its maximum activity at temperatures above $60{ }^{\circ} \mathrm{C}$. The rapid decomposition of hydroxylamine at higher temperatures prohibited activity measurements above $80{ }^{\circ} \mathrm{C}$.

XoxF-Type MDH Is Inhibited by Hydroxylamine. M. fumariolicum SolV encodes for a lanthanide-dependent XoxF-type $\mathrm{MDH}$, which was 
Table 1. Kinetic parameters of Methylacidiphilum fumariolicum SolV HAO (mHAO) compared to its characterized homologs from Kuenenia stuttgartiensis (KsHAO) and Nitrosomonas europaea (NeHAO)

\begin{tabular}{lccr} 
Enzyme & $\mathrm{mHAO}^{*}$ & $\mathrm{KsHAO}^{+}$ & $\mathrm{NeHAO}^{\ddagger}$ \\
\hline$V_{\text {max }} \mu \mathrm{mol} \mathrm{NH}{ }_{2} \mathrm{OH} \cdot \mathrm{min}^{-1} \cdot \mathrm{mg} \mathrm{HAO}^{-1}$ & 5.5 & 4.8 & 9.5 \\
$K_{\mathrm{m},} \mu \mathrm{M}$ & 1.4 & 4.4 & 3.6 \\
$K_{\text {cat }} \mathrm{s}^{-1}$ & 7.2 & 4.9 & 10.5 \\
$K_{\text {cat }} / K_{\mathrm{m}}, \mathrm{s}^{-1} \cdot \mu \mathrm{M}^{-1}$ & 5.1 & 1.1 & 2.9 \\
\hline
\end{tabular}

${ }^{*} \mathrm{pH} 7.5,60^{\circ} \mathrm{C}$.

${ }^{\dagger} \mathrm{pH} 7.0,37^{\circ} \mathrm{C}$; values calculated from data presented by Maalcke et al. (27). ${ }^{\ddagger} \mathrm{pH} 9.5$, room temperature; values calculated from data presented by Hooper and Nason (22).

purified directly from native biomass according to Pol et al. (41). To determine whether this enzyme is inhibited by hydroxylamine, as observed for the calcium-dependent MDH (30), methanol oxidation activity was assayed with various concentrations of hydroxylamine (0 to $100 \mu \mathrm{M})($ SI Appendix, Fig. S3). Hydroxylamine was found to be a potent inhibitor $\left(K_{\mathrm{i}}=0.52 \pm 0.3 \mu \mathrm{M}\right)$ of methanol oxidation by $M$. fumariolicum SolV XoxF-type MDH

\section{Discussion}

Aerobic and nitrite-dependent methanotrophs cometabolize ammonia and produce hydroxylamine. This is similar to aerobic ammonia-oxidizing microorganisms, which encode HAO proteins to further oxidize hydroxylamine to NO. Likewise, methanotrophs require a pathway to oxidize hydroxylamine, since it has been shown to be a potent inhibitor of calcium-dependent MDH $\left(K_{\mathrm{i}}=12 \mu \mathrm{M}\right)(30)$. Here, we showed that the lanthanidedependent MDH encoded by $M$. fumariolicum SolV was severely inhibited by hydroxylamine as well, with an inhibition constant of $K_{\mathrm{i}}=0.5 \mu \mathrm{M}$. Recent environmental surveys revealed that lanthanidedependent MDHs are prevalent and widespread in nature compared to the calcium-dependent MDHs (42). Consequently, the inhibition of lanthanide-dependent MDHs by hydroxylamine has an extensive impact, affecting environmentally relevant aerobic and nitrite-dependent methane oxidizers, which are both oxygen dependent $(10,43,44)$, and highlighting the importance of $\mathrm{HAO}$ in these methanotrophs.

Based on the transcriptional up-regulation of hao-like genes in response to ammonia, and the susceptibility of methanotrophic strains lacking hao genes to ammonia, it has been postulated that HAO-like proteins are also involved in hydroxylamine oxidation in methanotrophs, although biochemical evidence for this is lacking $(31,32,34)$. Here, we purified an HAO-like protein from the aerobic methanotroph $M$. fumariolicum SolV. Similar to other bona fide HAOs $(22,25-27,29)$, mHAO contained a $\mathrm{P}_{460}$ chromophore, characteristic of HAOs involved in oxidative reactions (28). mHAO oxidized hydroxylamine rapidly $\left(V_{\max }=\right.$ $5.5 \mu \mathrm{mol} \cdot \mathrm{min}^{-1} \cdot \mathrm{mg}$ protein $\left.{ }^{-1}, K_{\mathrm{m}}=1.4 \mu \mathrm{M}, K_{\text {cat }}=7.2 \mathrm{~s}^{-1}\right)$ and produced NO, as was recently demonstrated for aerobic and anaerobic ammonia-oxidizing bacteria $(27,29)$. The mHAO kinetic parameters were in the same order of magnitude as those observed for previously characterized ammonia oxidizer HAOs $(22,27)$, indicating that mHAO was an efficient hydroxylamine-oxidizing enzyme. Considering all secondary structure elements were conserved among the different mHAO sequences, it is highly likely that mHAO is involved in hydroxylamine oxidation in all oxygendependent methanotrophs.

In aerobic ammonia-oxidizing bacteria, the concerted activity of ammonia monooxygenase (AMO) and HAO convert ammonia to NO. These microorganisms primarily oxidize the produced NO to nitrite contributing to energy conservation via a hitherto-unknown NO-oxidizing enzyme (24). Essentially, the same pathway also occurs in oxygen-dependent methanotrophs, where methane monooxygenase and mHAOs convert ammonia via hydroxylamine to NO. How the produced NO is further converted could differ in distinct aerobic and nitrite-dependent methanotrophs. Many isolated aerobic methanotrophs, similar to aerobic ammonia oxidizers, produce nitrite as the end product of their ammoniaoxidizing activity (31-35), where we now show that NO is also an obligate free intermediate. Consequently, both clades of microorganisms would require an NO-oxidizing enzyme. Although the exact nature of this enzyme remains unknown, it is tempting to speculate that such an enzyme could be another shared feature in the nitrogen metabolism of ammonia- and methane-oxidizing microorganisms. Using the set of proteins they encode for ammonia oxidation to nitrite, known aerobic methanotrophs would be unable to grow, since they lack the hydroxylamine-ubiquinone reduction module that would couple this oxidation activity to energy conservation (4). In contrast to most cultured aerobic methanotrophs, conversion of ammonia to $\mathrm{N}_{2} \mathrm{O}$ without external nitrite addition or detection of intermediary nitrite production has been shown in 14 strains of Methylomonas methanica, Methylomonas koyamae, and Methylomonas lenta (36). In these microorganisms, it is conceivable that instead of being first oxidized to nitrite, NO could be directly reduced to $\mathrm{N}_{2} \mathrm{O}$ by an $\mathrm{NO}$ reductase, which is encoded by many methanotrophs $(4,31)$. Next to bona fide aerobic methanotrophs, also nitrite-dependent methane-oxidizing bacteria encode mHAO proteins $(44,45)$. These microorganisms use NO to produce their own oxygen, which they then use to active methane (45). Here, ammonia oxidation could potentially serve as an alternative source of $\mathrm{NO}$, and could contribute to energy conservation and growth in nitrite-dependent methanotrophs.

Environmental ammonium concentrations up to $28 \mathrm{mM}$ and temperatures up to $70{ }^{\circ} \mathrm{C}$ are found in volcanic mud pots, which are the natural habitat of $M$. fumariolicum $(39,46)$. In agreement with this, mHAO of $M$. fumariolicum is very thermostable, maintaining $80 \%$ of its maximum activity to at least $80{ }^{\circ} \mathrm{C}$, compared to the HAOs of mesophilic ammonia oxidizers, which are nearly completely inactive above $60{ }^{\circ} \mathrm{C}(22)$. This enables $M$. fumariolicum to prevent hydroxylamine inhibition in its ammonia-rich natural environment, which lack an apparent bona fide ammonia-oxidizing
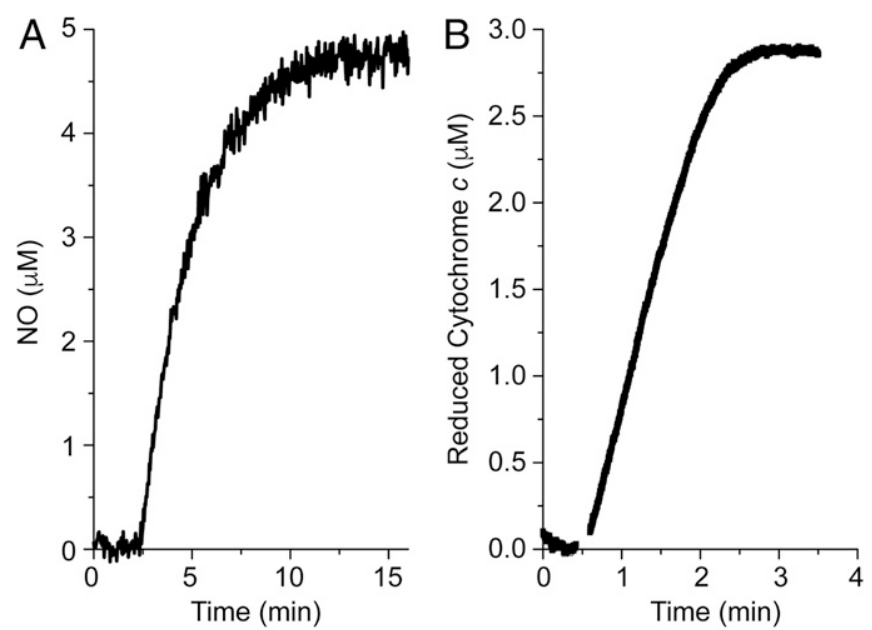

Fig. 2. NO production by $\mathrm{mHAO}$ from Methylacidiphilum fumariolicum SolV. (A) Sample trace of NO production by mHAO from $5 \mu \mathrm{M} \mathrm{NH} \mathrm{N}_{2} \mathrm{OH}$ measured with membrane inlet mass spectrometry (MIMS). Hydroxylamine oxidation assays by mHAO with varying concentrations of hydroxylamine ( 2 to $5 \mu \mathrm{M})$, resulting in a recovery rate of $94 \pm 4 \%(n=6)$ of NO compared to the added amount of hydroxylamine. (B) Sample trace of cytochrome $c$ reduction by $\mathrm{mHAO}$ with hydroxylamine. Addition of $1 \mu \mathrm{M}$ hydroxylamine resulted in the reduction of $3 \pm 0.06 \mu \mathrm{M}(n=3)$ cytochrome $c$, indicating the three-electron oxidation of hydroxylamine to $\mathrm{NO}$ by $\mathrm{mHAO}$. 


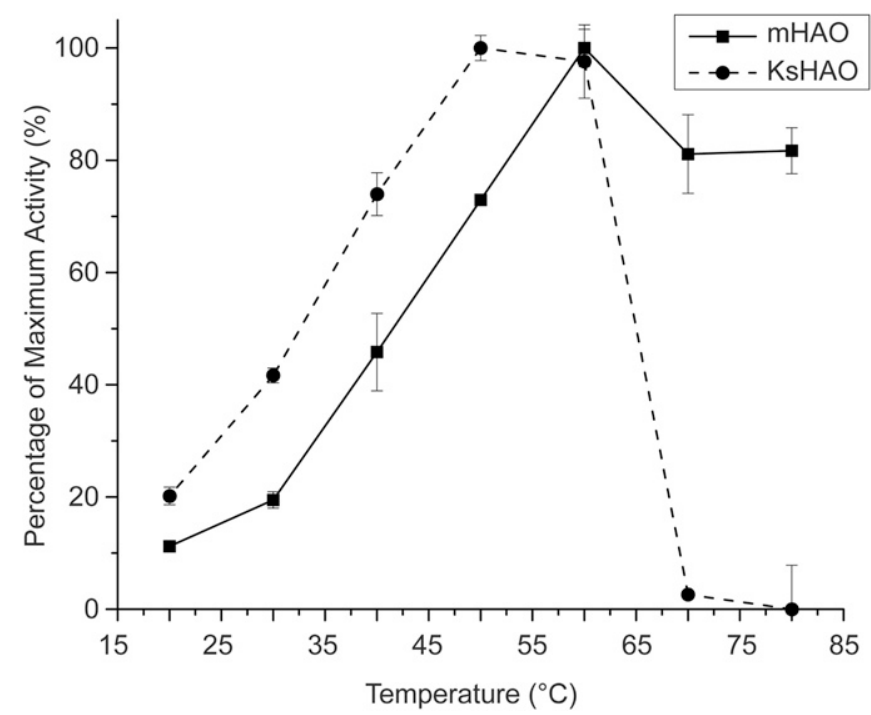

Fig. 3. Temperature dependence of mHAO from Methylacidiphilum fumariolicum SolV (solid) and KsHAO from Kuenenia stuttgartiensis (dashed) expressed as a percentage of the maximum activity ( $n=3$ per temperature; bars represent SD). The temperature dependence for $\mathrm{mHAO}$ is shifted by $10{ }^{\circ} \mathrm{C}$ compared to KsHAO. Activity of $\mathrm{KsHAO}$ is almost completely abolished at temperatures above $60{ }^{\circ} \mathrm{C}$, whereas mHAO retains $80 \%$ of its activity at these temperatures.

microorganism. Next to volcanic mud pots, oxygen-dependent methane oxidizers commonly occur in other methane- and ammonia-rich ecosystems such as freshwater lakes, marine sediments, rice paddy fields, and landfills (7, 8, 13-15, 17, 43, 47, 48). In such environments, the multiheme mHAO, which is encoded by many methanotrophs, facilitates methane oxidizers to act as ammonia oxidizers.

\section{Materials and Methods}

Unless otherwise noted, all chemicals were purchased from VWR Chemicals (VWR International).

Growth of Methylacidiphilum fumariolicum SolV. Methylacidiphilum fumariolicum SolV (39) used in this study was cultured in a 10-L chemostat (Applikon). The growth medium, previously described by Pol et al. (41), was supplemented with $200 \mathrm{mM}$ methanol. Methane was provided at a flow rate of $56 \mathrm{~mL} \cdot \mathrm{min}^{-1}$ via a mass flow controller. The reactor was kept at an oxygen concentration of $\sim 1 \%$ by adjusting the flow rate of air via a mass flow controller in a feedback loop, using an oxygen probe connected to a Biocontroller (Applikon). After an $\mathrm{OD}_{600}$ of 1 was obtained, the $\mathrm{pH}$ of the reactor was gradually increased from $\mathrm{pH} 2.7$ to $\mathrm{pH} 5$ through the stepwise addition of $2 \mathrm{M} \mathrm{NH}_{3}$, resulting in a final $\mathrm{NH}_{4}{ }^{+}$concentration of $20 \mathrm{mM}$ in the reactor. When the reactor reached a stable state at an $\mathrm{OD}_{600}$ of 5 , a $6 \mathrm{~mL} \cdot \mathrm{min}^{-1}$ bleed was started, which was collected in a refrigerated bottle at $4{ }^{\circ} \mathrm{C}$. Harvested cells were concentrated, washed once with ultrapure water (Merck-Millipore), and stored at $-20^{\circ} \mathrm{C}$.

mHAO Purification. Frozen cell pellets were thawed and resuspended in $20 \mathrm{mM}$ potassium phosphate buffer, $\mathrm{pH}$ 7. After breaking the cells by passing them three times through a French press (American Instrument Company) at 20,000 psi, cell debris and unbroken cells were removed by centrifugation at $10,000 \times g, 4{ }^{\circ} \mathrm{C}$ for $1 \mathrm{~h}$ (Sorvall LYNX 4000, F12-6 $\times 500$ LEX Fiberlite rotor; Thermo Fisher Scientific). The resulting supernatant was subjected to ultracentrifugation (Optima XE90; Beckman Coulter) at $160,000 \times g, 4{ }^{\circ} \mathrm{C}$, for $1 \mathrm{~h}$ in a fixed angle $45 \mathrm{Ti}$ rotor (Beckman Coulter) to separate the soluble and membrane fraction. The soluble fraction was loaded onto a SP Sepharose-FF column (XK26/20; GE Healthcare) equilibrated with $20 \mathrm{mM}$ potassium phosphate, $1 \mathrm{mM}$ methanol, $\mathrm{pH} 7.2$, and after washing, proteins were eluted using a linear gradient from 0 to $500 \mathrm{mM} \mathrm{NaCl}$ in 7 column volumes. Fractions containing hydroxylamine oxidizing activity were pooled, concentrated using 100-kDa cutoff spin filters (Vivaspin; Sartorius Stedim Biotech), and loaded onto a Superdex 200 size-exclusion column (GE Healthcare) equilibrated in $20 \mathrm{mM}$ potassium phosphate, $1 \mathrm{mM}$ methanol, $200 \mathrm{mM} \mathrm{NaCl}$, $\mathrm{pH}$ 7.2. Fractions containing pure mHAO were pooled, concentrated, and stored at $-80{ }^{\circ} \mathrm{C}$ until use. HAO from the anammox bacterium Kuenenia stuttgartiensis (KsHAO) was purified according to Maalcke et al. (27). All column chromatography was performed on an Äkta explorer FPLC system (GE Healthcare).

Enzyme Assays. Hydroxylamine oxidation was assayed following the reduction of bovine heart cytochrome c (Sigma-Aldrich) spectroscopically at $550 \mathrm{~nm}$ $\left(\Delta \varepsilon_{550}=19.6 \mathrm{mM}^{-1} \cdot \mathrm{cm}^{-1}\right)$ using a Cary 60 spectrophotometer (Agilent) in a $1-\mathrm{cm}$ path length Suprasil quartz cuvette (Hellma). Assays were performed in $50 \mathrm{mM}$ sodium phosphate, $50 \mathrm{mM} \mathrm{NaCl}, \mathrm{pH} 7.5$, with $50 \mu \mathrm{M}$ cytochrome $c$ and 1 to $1,000 \mu \mathrm{M}$ hydroxylamine (Sigma-Aldrich). After establishing a baseline, the reaction was started through the addition of $3 \mathrm{nM} \mathrm{mHAO}$ or KsHAO. To determine the optimal temperature, a range of 20 to $80^{\circ} \mathrm{C}$ was investigated at $100 \mu \mathrm{M}$ hydroxylamine concentrations. To determine the kinetic parameters, the temperature was kept constant at the previously determined optimum of $60^{\circ} \mathrm{C}$, and hydroxylamine concentrations were varied between 1 and $1,000 \mu \mathrm{M}$ All measurements were performed in triplicate, and reaction rates were determined from the initial linear portion of the curve using the Cary $60 \mathrm{soft}$ ware. Kinetics were determined by fitting the initial reaction rates, corrected for chemical background rates, to Michaelis-Menten equations using Origin 9.1 (OriginLab). Specific activities were calculated based on the mHAO concentrations in the sample, calculated using the absorbance of the $\mathrm{P}_{460}\left(\Delta \varepsilon_{468}=\right.$ $58.7 \mathrm{mM}^{-1} \cdot \mathrm{cm}^{-1}$ ). To determine the electron stoichiometry of hydroxylamine oxidation, the reaction was followed to completion and the amount of reduced cytochrome $c$ per mole hydroxylamine was calculated (27).

$\mathrm{MDH}$ activity assays were performed in duplicates using $20 \mathrm{mM}$ potassium phosphate, $\mathrm{pH} 7.2$, with $1 \mathrm{mM} \mathrm{KCN}, 1 \mathrm{mM}$ phenazine ethosulfate (Sigma-Aldrich), $200 \mu \mathrm{M}$ 2,6-dichlorophenolindophenol (DCPIP) (Sigma-Aldrich), 0 to $100 \mu \mathrm{M}$ methanol (HPLC grade; JT-Baker), and 0 to $100 \mu \mathrm{M}$ hydroxylamine at $45{ }^{\circ} \mathrm{C}$ Methanol oxidation was followed by measuring the reduction of DCPIP spectroscopically at $600 \mathrm{~nm}\left(\varepsilon_{600}=18.5 \mathrm{mM}^{-1} \cdot \mathrm{cm}^{-1}\right)$ in a SpectraMax 190 plate reader (Molecular Devices). After establishing a stable baseline, reactions were started by the addition of $1 \mu \mathrm{M}$ La-dependent MDH purified as described before (41). Kinetic parameters and the inhibition constant for hydroxylamine were determined by fitting the initial rates to a competitive enzyme-inhibition model using Origin 9.1 (OriginLab).

Membrane Inlet MS. Liquid concentrations of NO were measured with a Membrane Inlet Mass Spectrometer (HPR40, positive ion counting detector; Hiden Analytical) in a $10-\mathrm{mL}$ chamber. Setup of the MIMS, MIMS chamber, and MIMS probe were done as described previously by Schmitz et al. (49) Assays were performed in $10 \mathrm{mM}$ Hepes, $\mathrm{pH} 7$, with $100 \mu \mathrm{M}$ phenazine methosulfate (PMS) as electron acceptor and 2 to $5 \mu \mathrm{M}$ hydroxylamine at $45^{\circ} \mathrm{C}$. The NO signal was calibrated by flushing the liquid using a $4 \%$ NO in He gas bottle with a flow of $10 \mathrm{~mL} / \mathrm{min}$.

UV-Vis Spectroscopy. UV-vis absorbance spectra in the range of 350 to $600 \mathrm{~nm}$ were measured in a 1-cm path length Suprasil quartz cuvette (Hellma) using a Cary 60 spectrophotometer (Agilent) in an anaerobic glove box. After recording an as-isolated spectrum (fully oxidized), protein was reduced by the addition of a few grains of sodium dithionite and another spectrum was recorded. The ratio of $P_{460}$ to $550 \mathrm{~nm}$ varied between preparations. Since the $\mathrm{P}_{460}$ optical feature reports the active HAO population, the amount of $\mathrm{HAO}$ in the different preparations was determined using the extinction coefficient of the $\mathrm{P}_{460}\left(\Delta \varepsilon_{468}=58.7 \mathrm{mM}^{-1} \cdot \mathrm{cm}^{-1}\right)$, determined based on KsHAO (27).

Gel Electrophoresis. To assess the purity of mHAO during purification, polyacrylamide gel electrophoresis was routinely applied. Acrylamide gradient gels ( 4 to $15 \%$ ) were cast using a model 475 gradient delivery system (Bio-Rad). Denaturing gels were run and stained for protein and heme as adapted from previously described protocols $(50,51)$ using a Precision Plus Protein Dual Color standard (Bio-Rad) for molecular mass estimation. HrCN-1 gels were run according to the protocol described by Wittig et al. (52) using NativeMark Unstained protein standard (Thermo Fisher Scientific) for molecular mass estimation. Afterward, the gel was stained with Coomassie Brilliant Blue or with an in-gel hydroxylamine oxidation stain. The gel was incubated for $10 \mathrm{~min}$ in $20 \mathrm{mM}$ potassium phosphate, $\mathrm{pH} \mathrm{7,} \mathrm{with} 1 \mathrm{mM}$ nitro blue tetrazolium (Sigma-Aldrich), after which $100 \mu \mathrm{M}$ hydroxylamine was added. The gel was incubated at $50{ }^{\circ} \mathrm{C}$ until the stain was developed and then washed three times with ultrapure water.

MALDI-TOF MS. To identify proteins, MALDI-TOF MS analysis of tryptically digested peptides was performed as previously described (53). Spectra in the range of 600 to $3,000 \mathrm{~m} / \mathrm{z}$ were collected using a Microflex LRF MALDI-TOF 
(Bruker Daltonic) and analyzed using the Mascot Peptide mass Fingerprint program against the Methylacidiphilum fumariolicum SolV protein database, with a peptide tolerance of $0.3 \mathrm{Da}$, allowance of one missed cleavage and methionine oxidation as a variable modification.

Sequence Analysis. HAO sequences of various methanotrophs, Kuenenia stuttgartiensis and Nitrosomonas europaea, were downloaded from National Center for Biotechnology Information. Prediction and cleavage of signal peptides were performed with SignalP 5.0 (54). Multiple sequence alignment using the MUSCLE algorithm (55) was performed with MEGA X (56).

1. G. Myhre et al., "Anthropogenic and natural radiative forcing" in Climate Change 2013-The Physical Science Basis, T. F. Stocker et al., Eds. (Cambridge University Press, Cambridge, UK, 2013), chap. 8, pp. 659-740.

2. M. Saunois et al., The global methane budget 2000-2012. Earth Syst. Sci. Data 8, 697-751 (2016).

3. A. Y. Kallistova, A. Y. Merkel, I. Y. Tarnovetskii, N. V. Pimenov, Methane formation and oxidation by prokaryotes. Microbiology 86, 671-691 (2017).

4. L. Y. Stein, M. G. Klotz, Nitrifying and denitrifying pathways of methanotrophic bacteria. Biochem. Soc. Trans. 39, 1826-1831 (2011).

5. A. R. Ravishankara, J. S. Daniel, R. W. Portmann, Nitrous oxide $\left(\mathrm{N}_{2} \mathrm{O}\right)$ : The dominant ozone-depleting substance emitted in the 21st century. Science 326, 123-125 (2009).

6. R. S. Hanson, T. E. Hanson, Methanotrophic bacteria. Microbiol. Rev. 60, 439-471 (1996)

7. P. L. Bodelier, H. J. Laanbroek, Nitrogen as a regulatory factor of methane oxidation in soils and sediments. FEMS Microbiol. Ecol. 47, 265-277 (2004).

8. P. L. E. Bodelier, A. K. Steenbergh, Interactions between methane and the nitrogen cycle in light of climate change. Curr. Opin. Environ. Sustain. 9-10, 26-36 (2014)

9. L. Y. Stein, R. Roy, P. F. Dunfield, "Aerobic methanotrophy and nitrification: Processes and connections" in eLS, J. Battista et al., Eds. (Wiley, Chichester, UK, 2012). Available at https:/ onlinelibrary.wiley.com/doi/full/10.1002/9780470015902.a0022213. Accessed 28 May 2020

10. L. Y. Stein, The long-term relationship between microbial metabolism and greenhouse gases. Trends Microbiol. 28, 500-511 (2020).

11. M. Monteiro, J. Séneca, C. Magalhães, The history of aerobic ammonia oxidizers: From the first discoveries to today. J. Microbiol. 52, 537-547 (2014).

12. H. Daims, S. Lücker, M. Wagner, A new perspective on microbes formerly known as nitrite-oxidizing bacteria. Trends Microbiol. 24, 699-712 (2016).

13. P. L. Bodelier, P. Frenzel, Contribution of methanotrophic and nitrifying bacteria to $\mathrm{CH}_{4}$ and $\mathrm{NH}_{4}{ }^{+}$oxidation in the rhizosphere of rice plants as determined by new methods of discrimination. Appl. Environ. Microbiol. 65, 1826-1833 (1999).

14. J. Im, S. W. Lee, L. Bodrossy, M. J. Barcelona, J. D. Semrau, Field application of nitrogen and phenylacetylene to mitigate greenhouse gas emissions from landfill cover soils: Effects on microbial community structure. Appl. Microbiol. Biotechnol. 89, 189-200 (2011).

15. S. W. Lee et al., Effect of nutrient and selective inhibitor amendments on methane oxidation, nitrous oxide production, and key gene presence and expression in landfill cover soils: Characterization of the role of methanotrophs, nitrifiers, and denitrifiers. Appl. Microbiol. Biotechnol. 85, 389-403 (2009).

16. K. W. Mandernack et al., The biogeochemical controls of $\mathrm{N}_{2} \mathrm{O}$ production and emis sion in landfill cover soils: The role of methanotrophs in the nitrogen cycle. Environ. Microbiol. 2, 298-309 (2000)

17. C. Bédard, R. Knowles, Physiology, biochemistry, and specific inhibitors of $\mathrm{CH}_{4}, \mathrm{NH}_{4}{ }^{+}$ and $\mathrm{CO}$ oxidation by methanotrophs and nitrifiers. Microbiol. Rev. 53, 68-84 (1989).

18. M. O. Ross, A. C. Rosenzweig, A tale of two methane monooxygenases. J. Biol. Inorg Chem. 22, 307-319 (2017)

19. A. B. Hooper, T. Vannelli, D. J. Bergmann, D. M. Arciero, Enzymology of the oxidation of ammonia to nitrite by bacteria. Antonie van Leeuwenhoek 71, 59-67 (1997).

20. L. Chistoserdova, Modularity of methylotrophy, revisited. Environ. Microbiol. 13, 2603-2622 (2011)

21. C. Anthony, "Methanol dehydrogenase, a PQQ-containing quinoprotein dehydrogenase" in Enzyme-Catalyzed Electron and Radical Transfer, A. Holzenburg, N. S. Scrutton, Eds. (Springer, 2000), pp. 73-117.

22. A. B. Hooper, A. Nason, Characterization of hydroxylamine-cytochrome $c$ reductase from the chemoautotrophs Nitrosomonas europaea and Nitrosocystis oceanus. J. Biol. Chem. 240, 4044-4057 (1965).

23. L. E. Lehtovirta-Morley, Ammonia oxidation: Ecology, physiology, biochemistry and why they must all come together. FEMS Microbiol. Lett. 365, fny058 (2018)

24. K. M. Lancaster, J. D. Caranto, S. H. Majer, M. A. Smith, Alternative bioenergy: Updates to and challenges in nitrification metalloenzymology. Joule 2, 421-441 (2018).

25. A. B. Hooper, K. R. Terry, Hydroxylamine oxidoreductase of Nitrosomonas. Production of nitric oxide from hydroxylamine. Biochim. Biophys. Acta 571, 12-20 (1979).

26. P. Cedervall, A. B. Hooper, C. M. Wilmot, Structural studies of hydroxylamine oxidoreductase reveal a unique heme cofactor and a previously unidentified interaction partner. Biochemistry 52, 6211-6218 (2013).

27. W. J. Maalcke et al., Structural basis of biological NO generation by octaheme oxidoreductases. J. Biol. Chem. 289, 1228-1242 (2014).

28. M. G. Klotz et al., Evolution of an octahaem cytochrome $c$ protein family that is key to aerobic and anaerobic ammonia oxidation by bacteria. Environ. Microbiol. 10, 3150-3163 (2008)
Data Availability Statement. All study data are included in the article and SI Appendix.

ACKNOWLEDGMENTS. W.V., J.R., and M.S.M.J. were supported by the European Research Council (ERC) Advanced Grant (AG) Ecomom 339880. W.V. was also supported by Netherlands Organization for Scientific Research (NWO) Grant 824.15.011 (to B.K.). L.v.N. was supported by NWO Grant VI.Vidi.192.001. H.J.M.O.d.C. was supported by ERC AG VOLCANO 669371. B.K. was supported by ERC Starting Grant GreenT 640422. We thank Christina Ferousi for providing KsHAO, and Bram Vekeman, Jana Milucka, and Katharina Ettwig for critically reading the manuscript.

29. J. D. Caranto, K. M. Lancaster, Nitric oxide is an obligate bacterial nitrification intermediate produced by hydroxylamine oxidoreductase. Proc. Natl. Acad. Sci. U.S.A. 114, 8217-8222 (2017)

30. J. A. Duine, J. Frank Jr., Studies on methanol dehydrogenase from Hyphomicrobium X. Isolation of an oxidized form of the enzyme. Biochem. J. 187, 213-219 (1980).

31. M. A. Campbell et al., Model of the molecular basis for hydroxylamine oxidation and nitrous oxide production in methanotrophic bacteria. FEMS Microbiol. Lett. 322, 82-89 (2011).

32. A. T. Poret-Peterson, J. E. Graham, J. Gulledge, M. G. Klotz, Transcription of nitrification genes by the methane-oxidizing bacterium, Methylococcus capsulatus strain Bath. ISME J. 2, 1213-1220 (2008).

33. G. Nyerges, L. Y. Stein, Ammonia cometabolism and product inhibition vary considerably among species of methanotrophic bacteria. FEMS Microbiol. Lett. 297, 131-136 (2009).

34. S. S. Mohammadi, A. Pol, T. van Alen, M. S. M. Jetten, H. J. M. Op den Camp, Ammonia oxidation and nitrite reduction in the verrucomicrobial methanotroph Methylacidiphilum fumariolicum SolV. Front. Microbiol. 8, 1901 (2017).

35. G. Nyerges, S. K. Han, L. Y. Stein, Effects of ammonium and nitrite on growth and competitive fitness of cultivated methanotrophic bacteria. Appl. Environ. Microbiol. 76, 5648-5651 (2010).

36. S. Hoefman et al., Niche differentiation in nitrogen metabolism among methanotrophs within an operational taxonomic unit. BMC Microbiol. 14, 83 (2014).

37. K. D. Kits, D. J. Campbell, A. R. Rosana, L. Y. Stein, Diverse electron sources support denitrification under hypoxia in the obligate methanotroph Methylomicrobium album strain BG8. Front. Microbiol. 6, 1072 (2015).

38. K. D. Kits, M. G. Klotz, L. Y. Stein, Methane oxidation coupled to nitrate reduction under hypoxia by the Gammaproteobacterium Methylomonas denitrificans, sp. nov. type strain FJG1. Environ. Microbiol. 17, 3219-3232 (2015).

39. A. Pol et al., Methanotrophy below pH 1 by a new Verrucomicrobia species. Nature 450, 874-878 (2007).

40. K. R. Terry, A. B. Hooper, Hydroxylamine oxidoreductase: A 20-heme, 200000 molecular weight cytochrome $c$ with unusual denaturation properties which forms a 63000 molecular weight monomer after heme removal. Biochemistry 20, 7026-7032 (1981).

41. A. Pol et al., Rare earth metals are essential for methanotrophic life in volcanic mudpots. Environ. Microbiol. 16, 255-264 (2014).

42. L. Chistoserdova, M. G. Kalyuzhnaya, Current trends in methylotrophy. Trends Microbiol. 26, 703-714 (2018).

43. J. S. Graf et al., Bloom of a denitrifying methanotroph, "Candidatus Methylomirabilis limnetica," in a deep stratified lake. Environ. Microbiol. 20, 2598-2614 (2018).

44. W. Versantvoort et al., Comparative genomics of Candidatus Methylomirabilis species and description of Ca. Methylomirabilis lanthanidiphila. Front. Microbiol. 9, 1672 (2018).

45. K. F. Ettwig et al., Nitrite-driven anaerobic methane oxidation by oxygenic bacteria. Nature 464, 543-548 (2010)

46. A. F. Khadem, A. Pol, M. S. M. Jetten, H. J. M. Op den Camp, Nitrogen fixation by the verrucomicrobial methanotroph "Methylacidiphilum fumariolicum" SolV. Microbiology 156, 1052-1059 (2010).

47. P. L. E. Bodelier, Interactions between nitrogenous fertilizers and methane cycling in wetland and upland soils. Curr. Opin. Environ. Sustain. 3, 379-388 (2011).

48. S. Acton, E. Baggs, Interactions between $\mathrm{N}$ application rate, $\mathrm{CH}_{4}$ oxidation and $\mathrm{N}_{2} \mathrm{O}$ production in soil. Biogeochemistry 103, 15-26 (2011).

49. R. A. Schmitz et al., The thermoacidophilic methanotroph Methylacidiphilum fumariolicum SolV oxidizes subatmospheric $\mathrm{H}_{2}$ with a high-affinity, membraneassociated [NiFe] hydrogenase. ISME J. 14, 1223-1232 (2020).

50. U. K. Laemmli, Cleavage of structural proteins during the assembly of the head of bacteriophage T4. Nature 227, 680-685 (1970).

51. D. D. Mruk, C. Y. Cheng, Enhanced chemiluminescence (ECL) for routine immunoblotting: An inexpensive alternative to commercially available kits. Spermatogenesis 1, 121-122 (2011).

52. I. Wittig, M. Karas, H. Schägger, High resolution clear native electrophoresis for in-gel functional assays and fluorescence studies of membrane protein complexes. Mol. Cell. Proteomics 6, 1215-1225 (2007).

53. M. H. Farhoud et al., Protein complexes in the archaeon Methanothermobacter thermautotrophicus analyzed by blue native/SDS-PAGE and mass spectrometry. Mol. Cell. Proteomics 4, 1653-1663 (2005).

54. J. J. Almagro Armenteros et al., SignalP 5.0 improves signal peptide predictions using deep neural networks. Nat. Biotechnol. 37, 420-423 (2019).

55. R. C. Edgar, MUSCLE: Multiple sequence alignment with high accuracy and high throughput. Nucleic Acids Res. 32, 1792-1797 (2004).

56. S. Kumar, G. Stecher, M. Li, C. Knyaz, K. Tamura, MEGA X: Molecular evolutionary genetics analysis across computing platforms. Mol. Biol. Evol. 35, 1547-1549 (2018). 